\title{
Grippe au Canada, saison 2012-2013
}

\author{
Wong E. ${ }^{1}$, Yan Li Y. ${ }^{2}$, Bastien N. ${ }^{2}$, Sevenhuysen C. ${ }^{1}$, Khalil N. ${ }^{1}$, \\ Winchester B. ${ }^{1}$, Saboui M. ${ }^{1}$, Mersereau T. ${ }^{1}$ \\ ${ }^{1}$ Centre de l'immunisation et des maladies respiratoires infectieuses, Agence de la santé publique du Canada, Ottawa \\ (Ontario) \\ ${ }^{2}$ Laboratoire national de microbiologie, Agence de la santé publique du Canada, Winnipeg (Manitoba) \\ Personne-ressource : fluwatch@phac-aspc.gc.ca
}

\section{Résumé}

Objectif : Le présent rapport résume l'activité grippale au Canada au cours de la saison de la grippe de 20122013 (du 26 août 2012 au 24 août 2013) à partir des données obtenues par l'entremise du programme de Surveillance de l'influenza.

Méthodes : Le programme de Surveillance de l'influenza a recueilli des données à partir de six principaux indicateurs d'activité grippale qui décrivent le comportement épidémiologique et virologique de la grippe au Canada : la détection de cas de grippe dans des laboratoires sentinelles; la caractérisation des souches et la résistance aux antiviraux des virus grippaux en circulation; les taux de consultation dans les cabinets de soins primaires pour un syndrome grippal; les niveaux régionaux d'activité grippale; les résultats graves associés à la grippe; et la surveillance pharmaceutique.

Résultats : La saison grippale a atteint son sommet à l'échelle nationale entre la fin décembre 2012 et le début janvier 2013 par le virus de la grippe $A(H 3 N 2)$ déterminé comme la souche prédominante de grippe en circulation jusqu'au début du mois de mars, lorsque le virus de la grippe $B$ est devenu la souche prédominante. Les taux d'hospitalisation cumulatifs déclarés pour tous les groupes d'âge étaient de 25,0 cas pour 100000 habitants. Le virus de la grippe A touche plus fortement les adultes âgés de 65 ans ou plus, tandis que la grippe $B$ touche plus fortement les enfants âgés de 19 ans ou moins.

Conclusion : La saison de la grippe a été assez grave. Comparativement aux deux saisons précédentes, considérées comme étant relativement bénignes, on a observé une augmentation importante du nombre de détections de la grippe en laboratoire, de même que du nombre d'hospitalisations associées à la grippe en 20122013.

\section{Introduction}

La grippe est une infection respiratoire généralement causée par les virus de la grippe A ou $B$. Au Canada, elle survient généralement chaque année, à la fin de l'automne et en hiver. L'infection grippale cause une maladie primaire, et elle peut aussi entraîner des complications médicales secondaires graves, y compris la pneumonie virale, la pneumonie bactérienne secondaire et l'aggravation de pathologies sous-jacentes. Au Canada, on estime une moyenne annuelle de 12200 hospitalisations causées par la grippe (1-3) et environ 3500 décès par année y sont attribuables (4).

Le programme de Surveillance de l'influenza du Centre de l'immunisation et des maladies respiratoires infectieuses (CIMRI) de l'Agence de la santé publique du Canada coordonne la surveillance nationale de la grippe. Les principaux objectifs de ce programme sont la détection précoce et la déclaration rapide de l'activité grippale au Canada et à l'étranger ainsi que la surveillance des souches du virus de la grippe en circulation, notamment la caractérisation antigénique, la détermination de nouveaux sous-types et les modifications de la résistance aux antiviraux. 
Le programme de Surveillance de l'influenza recueillait des données et des renseignements sur l'activité grippale à partir de diverses sources et les diffusait chaque semaine pendant la saison active de la grippe (de septembre à la mi-mai) et de façon bihebdomadaire durant la période de faible activité (de la mi-mai à août). Les professionnels de la santé et le public ont accès à ces renseignements par courriel et sur le site Web du programme de Surveillance de l'influenza : http://www.phac-aspc.gc.ca/fluwatch/index-fra.php.

Le présent rapport fournit un résumé épidémiologique et virologique de l'activité grippale au Canada au cours de la saison 2012-2013.

\section{Méthodes}

Le présent rapport est fondé sur les six principaux indicateurs d'activité grippale signalée chaque semaine partout au Canada du 26 août 2012 (semaine 35) au 24 août 2013 (semaine 34) : I) les détections de la grippe et d'autres virus respiratoires par les laboratoires sentinelles; II) la caractérisation des souches et la résistance aux antiviraux des virus grippaux en circulation; III) les taux de consultation dans des cabinets de soins primaires pour un syndrome grippal déclarés par les praticiens sentinelles; IV) les niveaux d'activité grippale à l'échelle régionale; V) les hospitalisations et les décès attribuables à la grippe; et VI) la surveillance pharmaceutique.

Ces ensembles de données ont été recueillis à l'aide de la surveillance continue de la santé publique, ils n'ont pas à être approuvés par un comité d'éthique de la recherche.

\section{Ensemble des données de surveillance}

(I) Détections de la grippe par les laboratoires sentinelles

Les cas détectés de grippe ont été signalés par l'intermédiaire du Programme de surveillance et de détection de virus des voies respiratoires (PSDVVR) des laboratoires sentinelles. Ils sont présentés de façon regroupée et sont accompagnés des détails sur les cas. Les laboratoires participants ont signalé le nombre total de tests réalisés pour la grippe et le nombre total de résultats positifs. Les échantillons des territoires sont testés par des laboratoires de référence de provinces voisines et regroupés en résultats provinciaux. Les échantillons associés à des données sur chaque cas étaient attribuables à un territoire et ont été déclarés indépendamment de la province qui a effectué les tests sur les échantillons.

(II) Caractérisation des souches et résistance aux antiviraux des virus grippaux en circulation

Le LNM a exercé une surveillance nationale des souches humaines de virus grippal en collaboration avec les laboratoires provinciaux et d'autres laboratoires canadiens en milieux hospitalier et universitaire. Un sousensemble des cas de virus grippaux détectés au Canada a été transmis chaque semaine au LNM pour y subir des tests supplémentaires servant à caractériser les souches et à évaluer les changements antigéniques de même que la résistance aux antiviraux dans les souches de virus grippal en circulation.

(III) Taux de consultation dans des cabinets de soins primaires pour un syndrome grippal déclarés par les praticiens sentinelles

Le CIMRI a recruté et géré la participation des médecins sentinelles dans sept provinces et trois territoires. Dans les trois autres provinces (Colombie-Britannique, Alberta et Saskatchewan), le recrutement des praticiens sentinelles et la déclaration des données ont été gérés par des programmes provinciaux indépendants. Pendant une journée de clinique chaque semaine, les praticiens sentinelles signalaient le nombre total de patients ayant consulté pour n'importe quelle raison (dénominateur) et le nombre total de patients correspondant à la définition de cas normalisée à l'échelle nationale du syndrome grippal (numérateur).

(IV) Niveaux régionaux d'activité grippale et éclosions régionales 
Les provinces et territoires ont été subdivisés en régions de surveillance. Les épidémiologistes provinciaux et territoriaux ont évalué le niveau d'activité grippale hebdomadaire sur leur territoire respectif, d'après le nombre de cas de grippe détectés en laboratoire, la présence de syndrome grippal et les rapports d'éclosions de grippe ou de syndrome grippal. II existait quatre catégories standard d'activité : aucune activité, activité sporadique, activité localisée et activité étendue. Les éclosions dans les hôpitaux et les établissements de soins de longue durée ont été déclarées toutes les semaines, et une éclosion a été définie comme deux cas de syndrome grippal ou plus au cours d'une période de sept jours comprenant au moins un cas de grippe confirmée en laboratoire.

\section{(V) Hospitalisations et décès attribuables à la grippe}

Les hospitalisations et les décès associés à des infections par le virus de la grippe confirmées en laboratoire ont été signalés par les provinces et territoires participants et deux réseaux de surveillance des hôpitaux. En 20122013, huit provinces et territoires (Alberta, Manitoba, Saskatchewan, Ontario, Île-du-Prince-Édouard, TerreNeuve-et-Labrador, les Territoires du Nord-Ouest et le Yukon) ont signalé des cas d'hospitalisation et de décès associés à la grippe dans leur province ou leur territoire. Le Programme canadien de surveillance active de l'immunisation (IMPACT) est un réseau d'hôpitaux pédiatriques de soins tertiaires qui comprend 12 centres situés dans huit provinces, ce qui représente environ $90 \%$ de tous les lits pédiatriques de soins tertiaires au pays. Le Réseau de surveillance des cas sévères (SOS) par le Réseau de recherche sur l'influenza de l'Agence de la santé publique du Canada et des Instituts de recherche en santé sur le Canada (PCIRN) se compose de 40 hôpitaux pour adultes dans six provinces, ce qui représente plus de 17000 lits de soins de courte durée pour adultes au Canada. Les hospitalisations et les décès signalés par les réseaux IMPACT et PCIRN peuvent également faire partie des déclarations provinciales et territoriales.

\section{(VI) Surveillance pharmaceutique - ventes de médicaments sur ordonnance}

Le système de surveillance pharmaceutique est coordonné par le Centre des maladies infectieuses d'origine alimentaire, environnementale et zoonotique (CMIOAEZ) à l'aide des données fournies par les pharmacies participantes à RxCanada. En 2012-2013, environ 2500 magasins de 15 grandes chaînes de pharmacies ont fourni des données en matière de médicaments sur ordonnance et en vente libre. Les pharmacies participantes étaient présentes dans toutes les provinces et tous les territoires à l'exception du Nunavut et dans $85 \%$ des régions sanitaires au Canada. Des données ont été recueillies sur les ordonnances nouvelles et renouvelées de médicaments achetés, qui sont classés dans la catégorie « antiviral » (y compris oseltamivir, zanamivir et amantadine) et « autre » (toutes les autres ordonnances).

\section{Résultats}

\section{Détections de la grippe et d'autres virus respiratoires par les laboratoires sentinelles}

Au cours de la saison 2012-2013, les laboratoires sentinelles ont testé 190376 échantillons pour la grippe et $31737(16,7 \%)$ se sont avérés positifs. Parmi ces résultats positifs, 27020 (85,1\%) étaient des cas de grippe A et 4717 (14,9\%) étaient des cas de grippe B. Parmi les virus de la grippe A saisonnière, 10669 (39,5\%) ont été sous-typés et 9395 (88,1\%) étaient des virus de la grippe $A(H 3 N 2)$ et 1274 (11,9\%) étaient des virus de la grippe $\mathrm{A}(\mathrm{H} 1 \mathrm{~N} 1)$ pdm09 (Figure 1). 
Figure 1 : Pourcentage de détections de cas de grippe en laboratoire au Canada, par type et soustype de virus grippal, 2012-2013
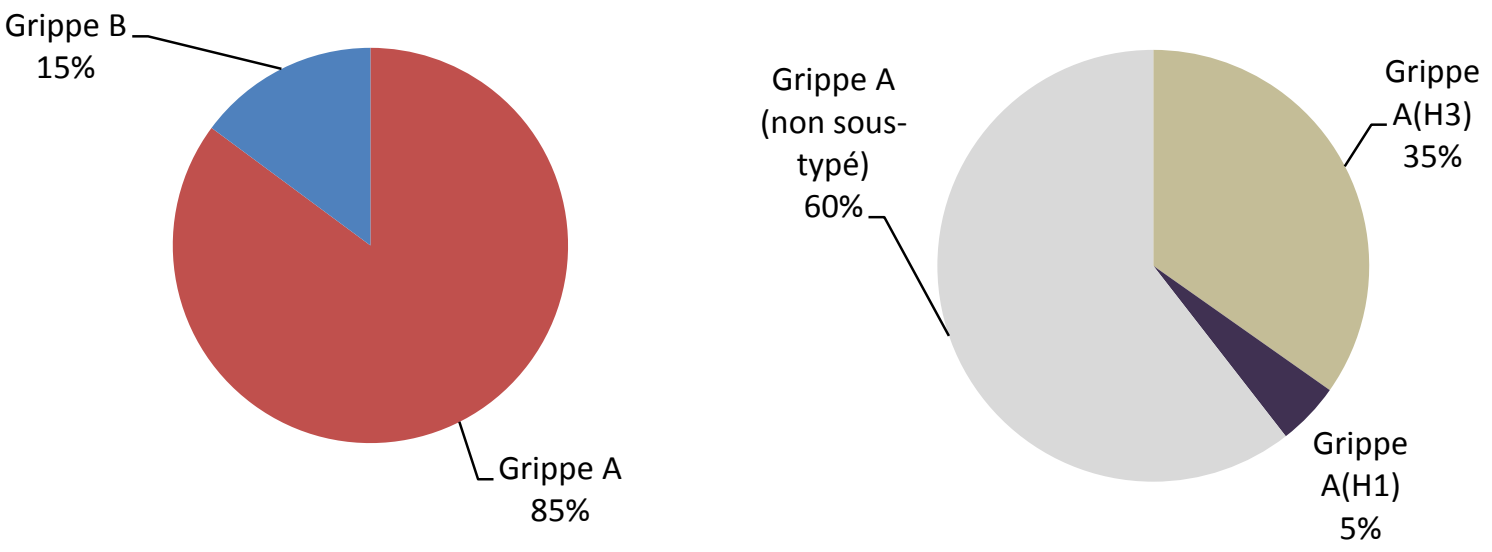

Selon les cas confirmés en laboratoire, la saison a atteint un sommet à l'échelle nationale entre la semaine 52 et la semaine 1 (du 23 décembre 2012 au 5 janvier 2013), période durant laquelle 35,0\% des échantillons provenant des voies respiratoires présentaient un résultat positif pour la grippe. La grippe $A(H 3 N 2)$ était la souche prédominante de grippe au cours de la saison 2012-2013. La grippe $A(H 3 N 2)$ était la souche prédominante jusqu'à la période entre la semaine 10 et la semaine 11 (du 3 au 16 mars 2013), durant laquelle la grippe $B$ est devenue la souche prédominante en circulation (Figure 2).

Figure 2: Pourcentage de détections de cas de grippe en laboratoire sentinelle au Canada, par semaine et type et sous-type de virus grippal, 2012-2013

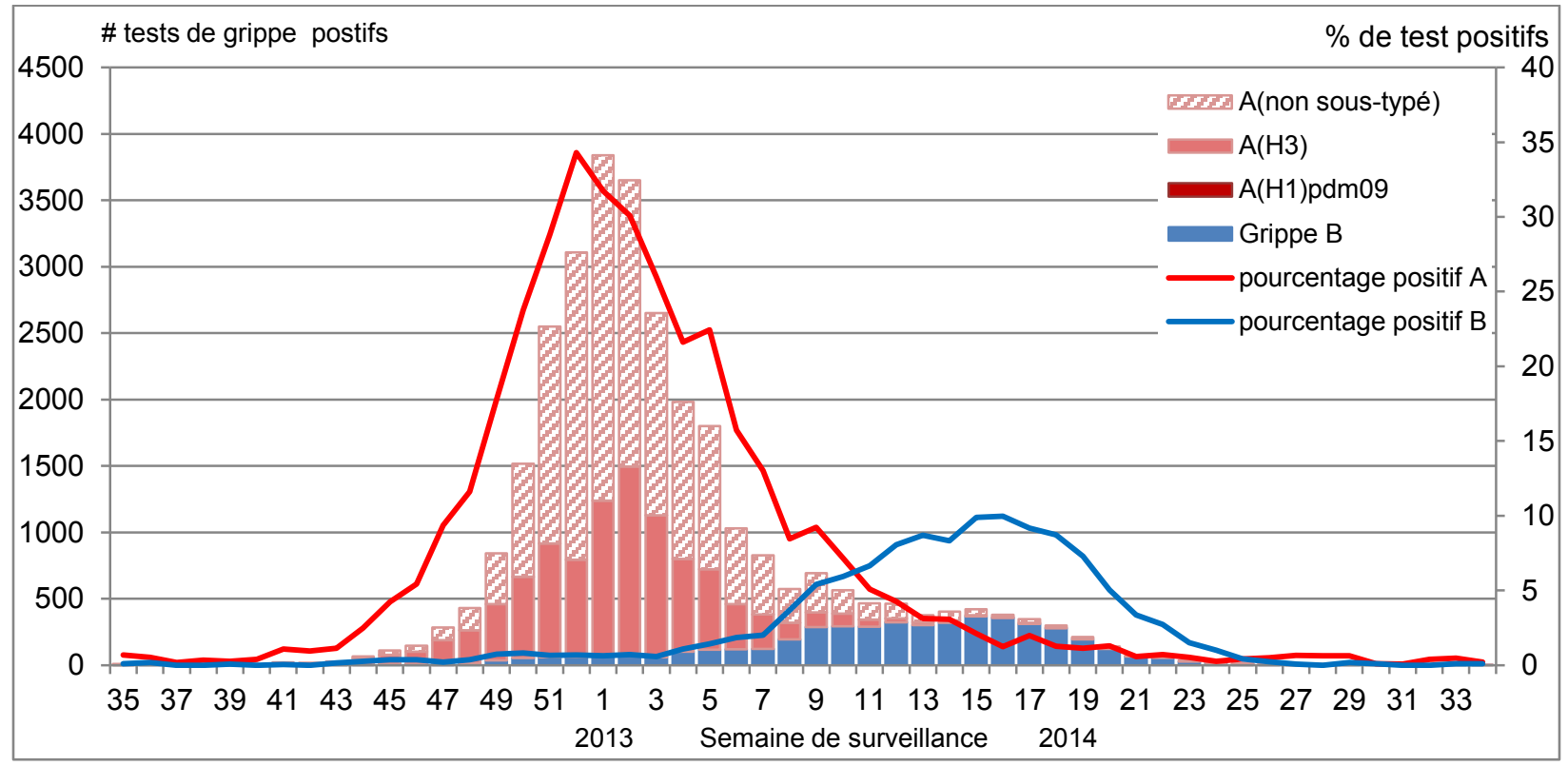


La proportion relative de chaque type et sous-type de virus en circulation et le moment où l'activité grippale a atteint un sommet variaient selon la région géographique. Le pourcentage d'échantillons positifs était le plus élevé au cours de la semaine 52 pour les régions de l'ouest et du centre du Canada (Colombie-Britannique, Alberta, Ontario et Québec) et Terre-Neuve-et-Labrador. La proportion de tests positifs était la plus élevée en Saskatchewan au cours de la semaine 2, au Manitoba au cours de la semaine 3 et dans les autres provinces de l'Atlantique au cours de la semaine 5 pour le Nouveau-Brunswick et de la semaine 6 pour l'île-du-Prince-Édouard et la Nouvelle-Écosse. La grippe A a été la souche prédominante dans toutes les provinces pour la saison, et la proportion de tests de laboratoire positifs pour la grippe $A$ a varié de 72,2 \% en Saskatchewan à 99,2 \% à l'île-duPrince-Édouard. À partir de données sur les cas, pour lesquels des données de laboratoire pour les territoires peuvent être déterminées, l'activité grippale semble avoir atteint son sommet au Nunavut au cours de la semaine 5 (du 27 janvier au 2 février 2013) et dans les Territoires du Nord-Ouest au cours de la semaine 17 (du 21 au 27 avril 2013). Aucune donnée n'est disponible pour le territoire du Yukon.

Parmi les 26546 cas pour lesquels on a obtenu des renseignements sur l'âge et le type/sous-type de virus cette saison, les adultes âgés de 65 ans ou plus représentaient $41,1 \%$ des cas de grippe (Tableau 1). Environ $45 \%$ des cas de grippe A se sont produits chez des adultes de 65 ans ou plus et $45,9 \%$ des cas de grippe B se sont produits chez les enfants de 19 ans ou moins.

Tableau 1 : Détections cumulatives des cas en laboratoire au Canada, par groupe d'âge, type ou soustype de virus, du 26 août 2012 au 24 août 2013

\begin{tabular}{|c|c|c|c|c|c|c|c|}
\hline \multirow{2}{*}{$\begin{array}{c}\text { Groupes } \\
\text { d'âge (ans) }\end{array}$} & \multicolumn{4}{|c|}{ Grippe A } & \multirow{2}{*}{$\begin{array}{c}\text { B } \\
\text { Total }\end{array}$} & \multicolumn{2}{|c|}{ Grippe A et B } \\
\hline & A Total & A(H1)pdm09 & $A(H 3)$ & $\begin{array}{l}\text { A(non } \\
\text { sous- } \\
\text { typé) }\end{array}$ & & $\mathbf{N}^{\text {bre }}$ & $\%$ \\
\hline$<5$ & 3040 & 224 & 847 & 1969 & 853 & 3893 & $14,7 \%$ \\
\hline $5-19$ & 1672 & 72 & 633 & 967 & 1081 & 2753 & $10,4 \%$ \\
\hline $20-44$ & 3568 & 359 & 1236 & 1973 & 731 & 4299 & $16,2 \%$ \\
\hline $45-64$ & 3766 & 331 & 1239 & 2196 & 703 & 4469 & $16,8 \%$ \\
\hline $65+$ & 10068 & 138 & 3767 & 6163 & 842 & 10910 & $41,1 \%$ \\
\hline Inconnu & 219 & 30 & 183 & 6 & 3 & 222 & $0,8 \%$ \\
\hline Total & 22333 & 1154 & 7905 & 13274 & 4213 & 26546 & $100 \%$ \\
\hline Pourcentage & $84,1 \%$ & $5,2 \%$ & $35,4 \%$ & $59,4 \%$ & $15,9 \%$ & & \\
\hline
\end{tabular}

\section{Caractérisation antigénique et résistance aux antiviraux}

Le Laboratoire national de microbiologie a caractérisé 1514 virus de la grippe au cours de la saison grippale 2012-2013. Les 662 virus de la grippe A(H3N2) étaient antigéniquement semblables à la souche du vaccin $A /$ Victoria/361/2011 et les 250 virus $A(H 1 N 1)$ pdm09 étaient antigéniquement semblables à la souche du vaccin $A / C a l i f o r n i a / 07 / 09$. Parmi les virus de la grippe $B, 464$ étaient antigéniquement semblables à la souche du vaccin B/Wisconsin/01/2010 (lignée Yamagata) et 138 étaient semblables à la souche B/Brisbane/60/2008 (lignée Victoria; composant du vaccin contre la grippe saisonnière de 2011-2012). 
Le Laboratoire national de microbiologie a soumis 1508 virus grippaux à des tests de résistance à l'oseltamivir, 1505 virus à des tests de résistance au zanamivir et 1344 virus de la grippe $A$ à des tests de résistance à l'amantadine. Des 653 virus de la grippe $\mathrm{A}(\mathrm{H} 3 \mathrm{~N} 2)$ testés, $1(0,2 \%)$ était résistant à l'oseltamivir et au zanamivir. Des 254 virus de la grippe $\mathrm{A}(\mathrm{H} 1 \mathrm{~N} 1)$ testés, $1(0,4 \%)$ était résistant à l'oseltamivir; tous étaient sensibles au zanamivir. Des 601 virus de la grippe B testés, $3(0,5 \%)$ étaient résistants à l'oseltamivir et au zanamivir. Tous les virus $\mathrm{A}(\mathrm{H} 3 \mathrm{~N} 2)$ sauf un étaient résistants à l'amantadine.

\section{Taux de consultation dans les cabinets de soins primaires pour un syndrome grippal (SG)}

Des 450 sentinelles ayant déclaré au moins un syndrome grippal au cours de la saison, $97 \%$ ont déclaré durant la saison active de la grippe (d'octobre à mai ou des semaines 40 à 20), dont $46 \%$ ont déclaré pendant au moins 15 des 33 semaines de la période active.

Le taux maximal de consultation pour le syndrome grippal était de 67,7 pour 1000 patients examinés et a été observé au cours de la semaine se terminant le 29 décembre 2012 (semaine 52), ce qui est semblable au pourcentage maximal de tests de laboratoire positifs pour la grippe (Figure 3). Les taux de consultations hebdomadaires pour un syndrome grippal ont dépassé l'écart de valeurs prévu, d'après les taux d'observation moyens des saisons précédentes depuis 1996-1997 (à l'exclusion des taux de la période de pandémie), au cours de la semaine 48 et entre les semaines 52 (2012) et 5 (2013). Les taux de consultation hebdomadaires pour un syndrome grippal sont demeurés égaux ou inférieurs à l'écart de valeurs prévu pour le reste de la saison.

Figure 3 : Taux de consultation pour le syndrome grippal (SG), par semaine de déclaration, comparé aux saisons 1996-1997 jusqu'à 2012-2013 (les données pour la pandémie ont été comprimées), Canada, 2012-2013

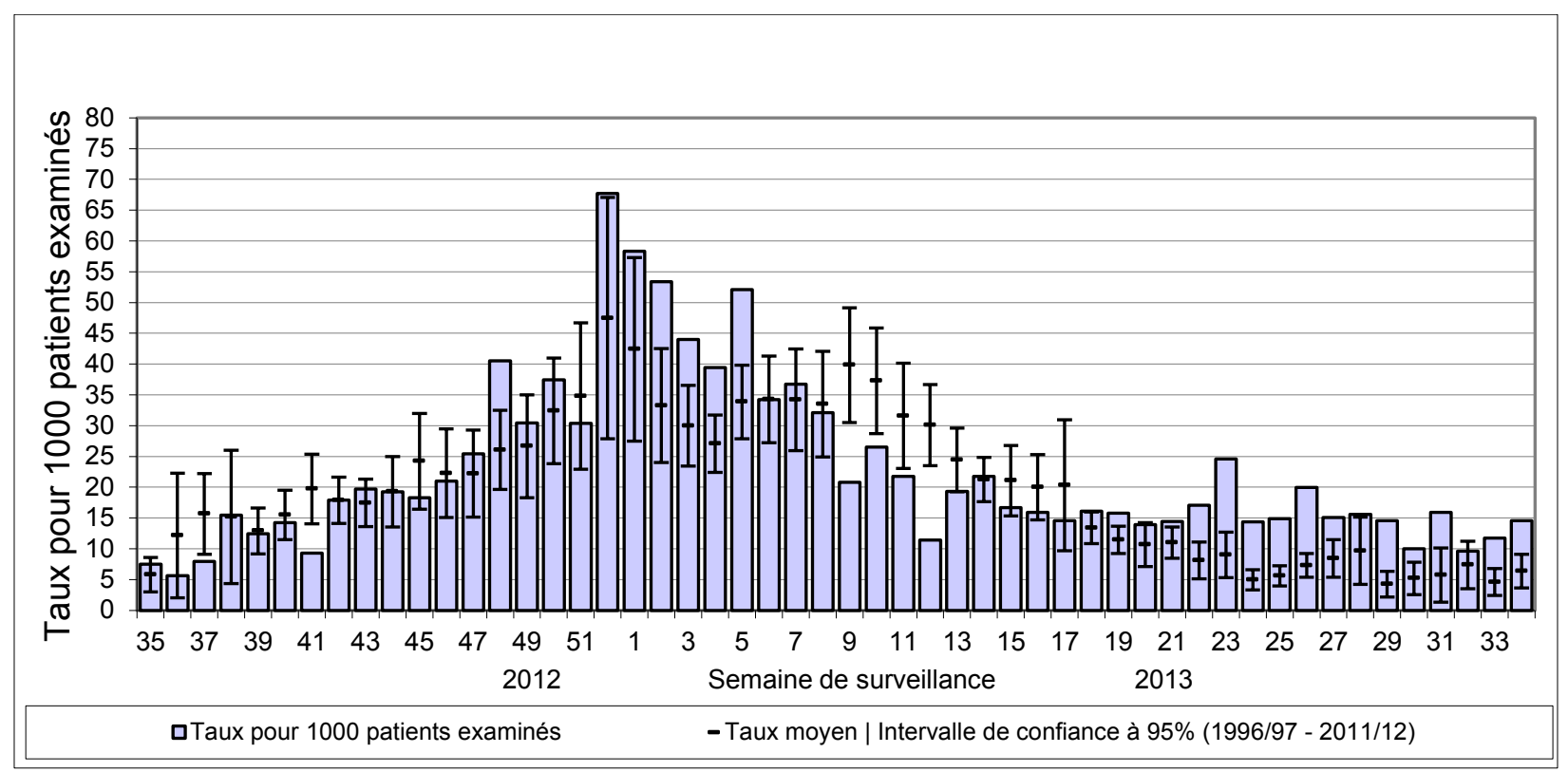

Les taux de consultation les plus élevés ont été signalés chez les enfants, avec une moyenne de 44,3 consultations pour un syndrome grippal pour 1000 patients examinés chez les 0 à 4 ans et de 46,2 consultations pour 1000 patients examinés chez les jeunes âgés de 5 à 19 ans au cours de la période active de la grippe (semaine 40 - semaine 20) durant la saison 2012-2013. 


\section{Niveaux régionaux d'activité grippale et éclosions régionales}

Les épidémiologistes provinciaux et territoriaux ont signalé la répartition géographique de la grippe dans leur province ou territoire. La répartition géographique de la grippe a été plus vaste au cours de la semaine se terminant le 12 janvier 2013 (semaine 2) pendant laquelle 14 régions ont signalé une activité étendue et 26 régions ont signalé une activité grippale localisée. La semaine se terminant le 15 juin 2013 (semaine 24) a été la première semaine durant laquelle aucune région n'a signalé d'activité grippale étendue ou localisée à la suite de la saison active de la grippe. Au cours de la saison, on a signalé 53 éclosions en milieu hospitalier et 676 éclosions dans des établissements de soins de longue durée, le nombre d'éclosions atteignant un sommet au cours de la semaine se terminant le 12 janvier 2013 (semaine 2).

\section{Résultats graves attribuables à la grippe (hospitalisations et décès)}

Au cours de la saison grippale 2012-2013, 5110 hospitalisations associées à la grippe ont été signalées par huit provinces et territoires. Les taux cumulatifs signalés (pour 100000 habitants) d'hospitalisations selon les groupes d'âge ont été calculés en fonction de la population des provinces et territoires participants. Les taux d'hospitalisation cumulatifs pour tous les groupes d'âge étaient de 25,0 cas pour 100000 habitants. Selon le groupe d'âge, les taux d'hospitalisation étaient de 66,4 (0 à 4 ans), 8,6 (5 à 19 ans), 6,5 (20 à 44 ans), 15,4 (45 à 64 ans) et 94,1 (65 ans et plus). Le taux de mortalité cumulatif était de 1,7 cas pour 100000 habitants.

En plus des signalements provinciaux et territoriaux, des cas ont également été signalés par deux réseaux de surveillance des hôpitaux. Le réseau IMPACT a signalé 889 hospitalisations pédiatriques et le réseau PCIRNSOS a signalé 1812 hospitalisations d'adultes. Une admission en unité de soins intensifs a été nécessaire pour $13 \%$ des hospitalisations pédiatriques et $12 \%$ des hospitalisations d'adultes (Figure 4). La grippe A était la souche prédominante déterminée dans les cas hospitalisés.

Figure 4. Pourcentage d'hospitalisations, d'admissions en unité de soins intensifs et de décès associés à la grippe, par groupe d'âge, Canada, 2012-2013

\section{a) Hospitalisations pédiatriques ( $\leq 16$ ans, IMPACT)}

\begin{tabular}{|c|c|c|c|c|c|c|c|c|c|c|c|}
\hline \multicolumn{4}{|c|}{ Proportion des cas } & $\square 0-5 m$ & \multicolumn{2}{|c|}{-6-23m } & \multicolumn{3}{|c|}{$5-9 a$} & \multicolumn{2}{|c|}{$\square$ 10-16a } \\
\hline & $0 \%$ & $10 \%$ & $20 \%$ & $30 \%$ & $40 \%$ & $50 \%$ & $60 \%$ & $70 \%$ & $80 \%$ & $90 \%$ & $100 \%$ \\
\hline Hospitalisations $(n=888)$ & & $18.9 \%$ & & $22.7 \%$ & & & $6 \%$ & & $21.3 \%$ & & $4 \%$ \\
\hline Admissions USI $(n=110)$ & 8.2 & & 29. & & & $27.3 \%$ & & 18 & & $17.3 \%$ & \\
\hline
\end{tabular}

b) Hospitalisations d'adultes ( $\geq 16$ ans, PCIRN-SOS)

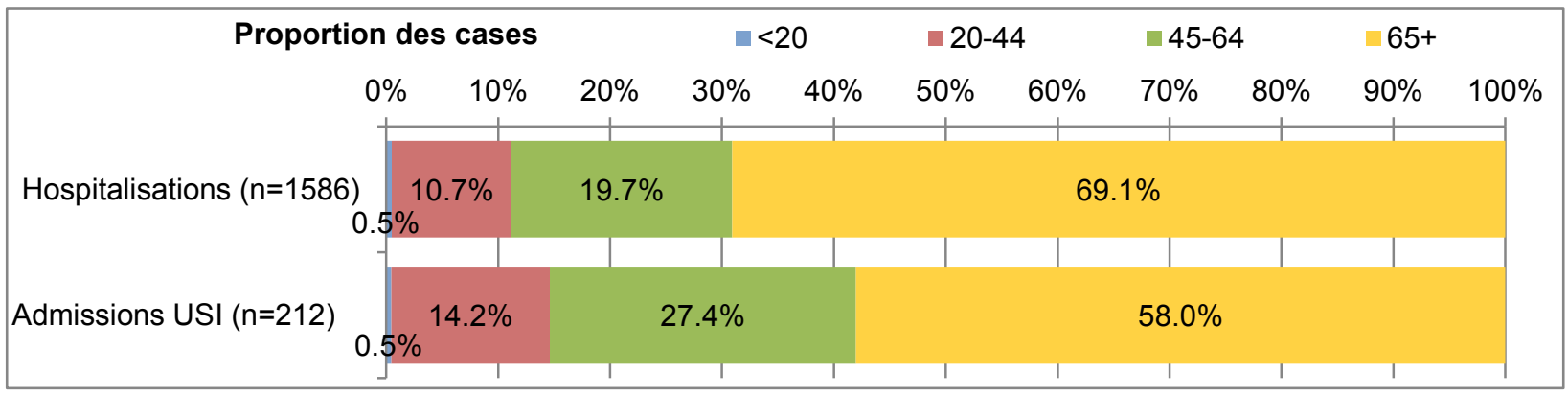


Parmi les 889 enfants hospitalisés, $70 \%$ ( $n=624)$ étaient âgés de moins de cinq ans, et $47 \%(n=419)$ étaient en bonne santé avant l'infection. Un total de 329 enfants hospitalisés présentait une affection sous-jacente pour laquelle la vaccination antigrippale est recommandée par le Comité consultatif national de l'immunisation (5). Un seul décès a été signalé chez les enfants.

Parmi les 1812 adultes hospitalisés, 68 \% ( $n=1230)$ étaient âgés de 65 ans ou plus, et 107 adultes sont décédés. Des 286 personnes admises à une unité de soins intensifs ou décédées, 122 présentaient des problèmes médicaux sous-jacents, 2 ne présentaient pas de problèmes médicaux sous-jacents et on ignorait les antécédents médicaux des autres personnes.

\section{Médicaments antiviraux - surveillance pharmaceutique}

Au Canada, le taux global d'ordonnances d'antiviraux a atteint un sommet au cours de la semaine 1 à 349 ordonnances d'antiviraux sur 100000 nouvelles ordonnances délivrées. Le taux d'ordonnances d'antiviraux était le plus élevé chez les personnes âgées de 65 ans ou plus et a atteint un sommet à 750 ordonnances sur 100000 ordonnances délivrées et il était plus faible chez les nourrissons (0-2 ans) et a atteint un sommet à 138 ordonnances sur 100000 ordonnances délivrées (Figure 5).

Figure 5 : Taux de ventes d'antiviraux contre la grippe sur ordonnance, par groupe d'âge et par semaine, Canada, 2012-2013

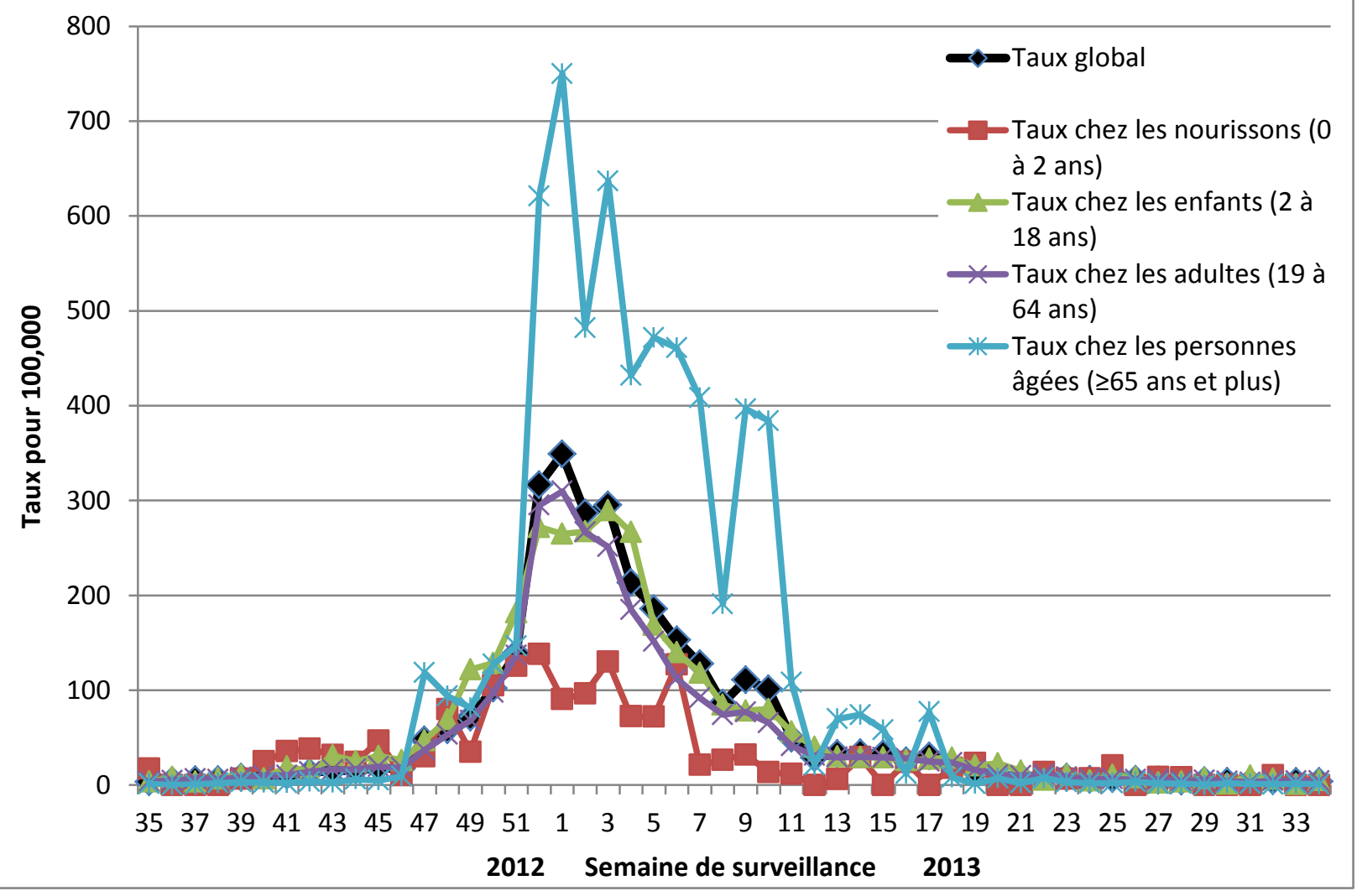




\section{Discussion}

Les divers indicateurs de surveillance de la grippe au cours de la saison 2012-2013 ont atteint un sommet de la fin de décembre 2012 au début de janvier 2013, ce qui est conforme au profil attendu de l'épidémie de grippe saisonnière. La grippe $A(H 3 N 2)$ était la souche de la grippe prédominante en circulation pour la saison et formait la majorité des cas de grippe jusqu'au début de mars 2013, lorsque la grippe B est devenue la souche prédominante en circulation.

La saison 2012-2013 a été assez grave comparativement aux deux saisons précédentes $(9,10)$. On a observé une augmentation des répercussions de la grippe dans le nombre de tests de laboratoire positifs pour la grippe signalés, avec près de deux fois le nombre de détections positives comparativement à celles de 2010-2011 et près de trois fois plus de détections qu'en 2011-2012. La même tendance a été observée au taux cumulatif d'hospitalisations et de décès signalés par les provinces et territoires participants. Le taux cumulatif d'hospitalisations et de décès pour 100000 habitants était de 14,6 et de 1,1 respectivement en 2010-2011 et de 8,5 et de 0,5 en 2011-2012 (données non publiées). Le nombre d'éclosions signalées cette saison dans les hôpitaux et les établissements de soins de longue durée était le plus élevé depuis 2004-2005.

Les tests de caractérisation et de résistance aux antiviraux par le Laboratoire national de microbiologie indiquent que les virus grippaux en circulation présentent peu de dérive antigénique et sont demeurés étroitement liés aux souches recommandées par l'OMS pour le vaccin trivalent contre la grippe en 2012-2013. Malgré cette concordance antigénique, certaines études ont signalé une efficacité faible à modérée du vaccin au cours de la saison 2012-2013 (6-8). Tous les virus testés sauf un faible pourcentage demeuraient sensibles aux inhibiteurs de la neuraminidase.

Un certain nombre de limites doivent être prises en compte lors de l'interprétation des résultats du programme de Surveillance de l'influenza. Les protocoles de dépistage en laboratoire, de surveillance et de déclaration ont varié avant, pendant et après la pandémie et ils ont également varié d'une province et d'un territoire à l'autre. Par conséquent, la comparaison des résultats de laboratoire ( $p$. ex. le pourcentage de résultats positifs et le nombre de cas confirmés en laboratoire) au fil du temps de même que les écarts observés entre les résultats des provinces et des territoires doivent être interprétés à la lumière de ces différences. Plusieurs facteurs influent sur le nombre d'isolats envoyés au Laboratoire national de microbiologie comme les limites de culture des isolats dans les laboratoires provinciaux et les échantillons d'intérêt clinique. Une plus grande proportion d'isolats précoces peut également être analysée afin de mieux comprendre les souches en circulation pour la saison à venir. Étant donné que seulement un sous-ensemble des virus de la grippe est caractérisé par le Laboratoire national de microbiologie, les données sur la répartition des souches ne sont pas représentatives des détections de grippe signalées par tous les laboratoires qui contribuent au Programme de surveillance et de détection de virus des voies respiratoires. Il est possible que les données propres à l'âge concernant le taux de consultation pour un syndrome grippal et les tests de dépistage de la grippe en laboratoire puissent être erronées par des biais liés à l'utilisation des soins de santé et le comportement des médecins qui effectuent les tests de dépistage. En outre, les taux de consultation pour un syndrome grippal peuvent varier au fil du temps et selon les régions en fonction de la participation des praticiens sentinelles, des différences entre les taux de couverture et de la cocirculation d'autres virus respiratoires. Le nombre réel d'individus touchés par la grippe au sein de la population n'est pas pris en compte dans les données étant donné que le programme de Surveillance de l'influenza s'appuie sur des sources de données sentinelles.

La double déclaration du nombre d'hospitalisations et de décès est possible là où une région compte un ou plusieurs hôpitaux participant au réseau IMPACT ou PCIRN-SOS. Les provinces et les territoires peuvent signaler une hospitalisation ou un décès sans que la raison ou la cause soient attribuables à la grippe. Les provinces et les territoires n'ont pas tous fourni des données d'ensemble sur les hospitalisations et les décès, de sorte que ces données sont incomplètes à l'échelle nationale. 
Les sources de données utilisées dans le cadre du programme Surveillance de l'influenza présentent certaines limites, cependant, lorsqu'on les regroupe, elles permettent de bien comprendre l'épidémiologie de la grippe au Canada pendant la saison grippale et d'effectuer des comparaisons entre les saisons. Le programme de Surveillance de l'influenza contribue toujours à renforcer la surveillance de la grippe au Canada en collaboration avec des partenaires qui prennent part à la surveillance à l'échelle nationale et internationale.

\section{Remerciements}

Les auteurs désirent transmettre leurs remerciements sincères aux partenaires du programme de Surveillance de l'influenza, qui ont participé au programme de Surveillance de l'influenza au cours de la saison grippale de 20122013, y compris les autorités sanitaires provinciales et territoriales, les médecins et les laboratoires sentinelles, le réseau IMPACT, le réseau PCIRN-SOS, le LNM et le CMIOAEZ.

\section{Conflit d'intérêts}

II n'y a aucun conflit d'intérêts à déclarer.

\section{Financement}

L'Agence de la santé publique du Canada, avec le soutien de contributions en nature par les partenaires du programme de Surveillance de l'influence, fournit le financement pour le programme de Surveillance de l'influenza.

\section{Références}

(1) Schanzer DL, McGeer A, Morris K. Statistical estimates of respiratory admissions attributable to seasonal and pandemic influenza for Canada. Influenza and other Respiratory Viruses. 2013; 7(5):799-808.

(2) Schanzer D, Langley J, Tam T. Role of influenza and other respiratory viruses in admissions of adults to Canadian hospitals. Influenza \& Other Respiratory Viruses 2008; 2(1):1-8.

(3) Schanzer DL, Langley JM, Tam TW. Hospitalization attributable to influenza and other viral respiratory illnesses in Canadian children. Pediatr Infect Dis J 2006; 25(0891-3668; 0891-3668; 9):795-800.

(4) Schanzer DL, Sevenhuysen C, Winchester B, et al. Estimating influenza deaths in Canada, 1992-2009. PLoS ONE. 2013; 8(11): e80481. doi:10.1371/journal.pone.0080481.

(5) Comité consultatif national de l'immunisation. Déclaration sur la vaccination antigrippale pour la saison $2012-2013$. Relevé des maladies transmissibles au Canada 2012; 38(DCC-2), août 2012.

(6) Skowronski DM, Janjua NZ, De Serres G, et al. Interim estimates of influenza vaccine effectiveness in 2012/13 from Canada's sentinel surveillance network, January 2013. Euro Surveill. 2013; 18(5):pii=20394. Accès : http://www.eurosurveillance.org/ViewArticle.aspx?Articleld=20394

(7) Skowronski DM, Janjua NZ, De Serres G, et al. Low 2012-13 influenza vaccine effectiveness associated with mutation in the Egg-Adapted H3N2 vaccine strain not antigenic drift in circulating viruses. PLoS ONE. 2014; 9(3):e92153. doi:10.1371/journal.pone.0092153.

(8) Kissling E, Valenciano M, Buchholz U, et al. Influenza vaccine effectiveness estimates in Europe in a season with three influenza type/subtypes circulating: the I-MOVE multicentre case-control study, influenza season 2012/13. Euro Surveill. 2014; 19(6):pii=20701. Accès : http://www.eurosurveillance.org/ViewArticle.aspx?Articleld=20701

(9) Agence de la santé publique du Canada. Influenza in Canada: 2010-11 season, par Domingo F, Winchester B, Aziz, S, et al. Ottawa: Agence de la santé publique du Canada, 2013. (Catalogue $n^{\circ}$ HP40-87/2013E-PDF).

(10) Agence de la santé publique du Canada. Influenza in Canada: 2011-12 season, par Wong E, Winchester B, Khalil N, et al. Ottawa: Agence de la santé publique du Canada, 2014. (Catalogue n HP37-18/2012E-PDF). 